\title{
Liposuction: Where are We and Where are We Going?
}

\section{Alethia Rubio Pena*}

Department of Plastic and Reconstructive Surgery, Centro Medico ISSEMYM, Toluca, Mexico

*Corresponding author: Alethia Rubio Pena, Department of Plastic and Reconstructive Surgery, Centro Medico ISSEMYM, Toluca, Mexico, E-mail: rubioalethia@gmail.com

\section{Received date: March 17, 2015, Accepted date: April 22, 2015, Published date: April 27, 2015}

Copyright: $(2015$ Alethia RP. This is an open-access article distributed under the terms of the Creative Commons Attribution License, which permits unrestricted use, distribution, and reproduction in any medium, provided the original author and source are credited.

Keywords: Liposuction; Anesthesiologists; Lipedema; Gynecomastia

\section{Introduction}

Liposuction is one of the most popular treatment modalities in aesthetic surgery around the World, counting 341,000 liposuction procedures just in 2008 and it was ranked second among all invasive cosmetic procedures, according to the American Society for Aesthetic Plastic Surgery [1]. The first surgical procedure was performed by Dujarrier in 1921. He used a uterine curette to remove fat from the knees of a well-known ballerina, with a disastrous outcome. In 1978 Kesselring added strong suction to this sharp curettage method. Shortly after, Illouz replaced the curette by a blunt cannula inserted subcutaneously and connected to a vacuum pump to aspirate the fatty tissues.

In the past decade, many innovations have been made and the anatomy and physiology of the fatty tissue have been studied in evergreater depth [2]. Several major advancements have also been contributed to the field of liposuction, including the introduction of the superwet and the tumescent wetting techniques, the refinement of cannulas for specific body sites, the utilization of some devices (Ultrasound-Assisted Aspiration, Vasser, Laser-Assisted Liposuction, Power Assisted Liposuction and Vibroliposuction) [3] and the use of manual syringe suction for fine contouring and autologous fat transfer.

\section{Patient Selection}

One of the most important aspects in the success of any surgical procedures is the physical condition of the patient at the time of surgery. Even though liposuction is generally an elective procedure, the liposuction patient must be assessed by the same standards as anyone who is undergoing any surgery including a complete preoperative history and physical examination [4].

Most liposuction procedures can be safely performed in an accredited office-based surgery facility or ambulatory surgery facility. Hospitalization may be required based on the patient's overall health area(s) of the body treated and the volume of aspirate removed5. In evaluating each patient, careful patient selection is extremely critical in large volume liposuction [4]. The body mass index (BMI) is a good method to assess the liposuction patient's relative risk/benefit for the procedure. In obese patients requiring large volume liposuction, it may be necessary to modify the anesthetic infiltrate solution to prevent lidocaine toxicity. Not all patients are appropriate liposuction candidates. These patients may wish to continue diet and exercise routines, seek medical intervention to treat existing condition(s), or, in the case of patients who have unrealistic expectations about their condition or potential outcomes, be referred for a psychiatric/ psychological evaluation [5]. Preoperative patient evaluation includes a thorough history and physical examination. Patients should be in either American Society of Anesthesiologists (ASA) class I (healthy with no medical problems) or ASA class II (medical problems well controlled on medications). Patients with uncontrolled medical problems are not candidates for large volume liposuction [4].

Failure to detect underlying cardiovascular, pulmonary, renal, hepatic, or thyroid disease can lead to fatal complications. A patient with a history of sleep apnea is at increased risk of fatal complications during the postoperative period. Patients must have an adequate cardiopulmonary reserve to handle the large volumes of wetting solution that are typical with large volume liposuction. A personal or family history of coagulopathies or bruising tendencies should be elicited. If any concerns exist regarding the health of the patient, refer the patient to the anesthesiologist or the patient's primary care physician for evaluation $[4,5]$.

Over-the-counter and prescription diet medications should be discontinued at least 2-3 weeks prior to surgery. Preoperative laboratory tests or ECG abnormalities should be evaluated thoroughly by a specialist, if needed, prior to surgery [4].

Candidates for large volume liposuction should be in a healthy state both physically and mentally. Weight should be stable or decreasing with diet and exercise. Patients who are experiencing rapid or persistent weight gain should be started on a program of exercise and nutritional modification before being accepted as surgical candidates. Do not offer noncompliant or poorly motivated patients large volume liposuction as a sole means of reducing their weight [4].

Patients must have reasonable goals and expectations. Perfectionist patients rarely are happy with the surgical outcome and should be avoided. Failure to exclude these patients can lead to clinical and aesthetic disasters and unhappy patients. Offering elective aesthetic surgery to patients with body dysmorphic disorder or eating disorders should be avoided [5]. If the patient has reasonable expectations that agree with technically achievable results, the patient undergoing large volume liposuction tends to be very happy. The key is to address preexisting skin contour irregularities, asymmetries, skin laxities, and redundancies in helping the patient understand what kind of result will be obtained. In these cases, the possibility of secondary procedures and touch-up procedures should also be emphasized [4]

\section{Indications}

The fundamental premise of liposuction is aspiration of adipose cells over a large area through small incisions required for the introduction of the aspirating cannula [6]. Originally was designed to correct unaesthetic superficial and deep deposits of subcutaneous fat, it produces highly satisfactory silhouette contouring when performed by appropriately trained operators using properly selected technologies for well-selected patients and anatomical areas [7]. 
Page 2 of 11

Liposuction should be considered foremost as a surgical procedure to enhance body contouring and not as a surgical modality for weight loss [8]. This technique must be specially committed to lifestyle changes to achieve long-term results [9]. The best results are still obtained when treating moderate localized fat deposits in a normal weight patient which cannot be managed by diet and exercise $[6,7]$.

The key to success is the capacity of the skin to redrape on the new adipose tissue shape, in order to avoid surface irregularities and wrinkles due to skin excess. Although a smooth, young and tight skin is a desirable criterion in patient selection, patients with less elastic or older skin, skin wrinkling or multiple fine irregularities (cellulite) may also benefit from liposuction and more specifically from superficial liposuction inducing more skin retraction [7].

Liposuction is continuously evolving and its indications are rapidly expanding; it has become an essential adjunct technique to improve results of many other aesthetic procedures including cervicoplasty, reduction mammaplasty, abdominoplasty, brachioplasty, thigh lift and postbariatric body contouring [8]. At present, there seems to be an enormous potential for the application of liposuction techniques in ablative and reconstructive surgery, outside the realm of purely aesthetic procedure.

Liposuction has evolved to define a specific technique that could be advantageously used in greatly diverse pathologies and conditions for treatment like some of the following:

Lipedema: It is a relatively new addition to the list of medical indications for liposuction surgery, with enormous treatment potential. It has received significant attention in recent years. It is characterized by increased deposition and edematous changes in the subcutaneous adipose tissue, particularly in the thighs and lower legs. Patients tend to develop orthostatic leg edema, which seems to be due to a capillary permeability disorder.

As a result of the increasing dynamic insufficiency of lymphatic transport, lymphostatic edema develops in the advanced stages followed by transition to lymphedema (lipolymphedema). In the past 5 years, this condition has been increasingly treated using liposuction $[7,9,10]$.

Gynecomastia: It is defined as unilateral or bilateral enlargement of the male breast. In these cases, it must be established prior to treatment whether the patient has 'genuine' gynecomastia with enlargement of the mammary gland as an expression of a hormone disorder (e.g. due to a gonadal disorder) or pseudogynecomastia as a result of increased fat accumulation. Breast enlargement as a result of fat accumulation can be treated very successfully with liposuction. Here, in particular, the use of vibration lipolysis is an advantage which can make treatment of this area considerably easier $[7,10]$.

Cellulite: It is commonly known as 'orange-peel skin', is a nonpathological skin change, which occurs mainly on the buttocks and thighs, as a result of gender-specific architecture of the adipose tissue. It usually develops over time with increasing age, but with a relevant predisposition, it may also be seen in young girls. Fat removal with liposuction represents a treatment option for cellulite because of specific changes to the subcutaneous space. The tissue-tightening effect due to healing of the deeper layers of the adipose tissue has an indirect positive effect on the uppermost layer. This prevents the sinking of this layer when standing, which is part of the reason for the mattress phenomenon $[7,10]$.
Lipodystrophy syndrome: The causes of lipodystrophy can vary enormously. They range from rare congenital lipodystrophy syndromes (such as congenital diffuse lipomatosis), fat distribution disorders in the form of imperfect osteogenesis, hereditary forms with unclear etiology and varying rates of progression, to acquired lipodystrophy. In clinical practice, localized acquired lipodystrophy (lipoatrophia circumscripta) is the most common. In the case of partial lipodystrophy syndromes, localized wasting of the adipose tissue occurs in some areas. The more hypertrophic areas of remaining adipose tissue result in an unsightly irregular appearance of the affected areas. This can lead to acute psychological stress and increasing social isolation. Aspiration of the prominent areas and injection of the fat obtained into the atrophic areas can significantly improve the mismatch between dystrophic and hypertrophic areas. In the case of progressive lipodystrophy syndromes, there is a need for continuous treatment of newly affected areas $[7,10]$.

Lipomas: Lipomas are the most common benign connective-tissue tumor, can occur as solitary lipomas or as multiple lipomas, possibly with a familial predisposition. Extirpation of a lipoma leaves an indentation in the skin depending on its size, but recurrence in the same place is unlikely because of complete removal. Aspiration can never result in total removal, and hence recurrence is possible. In the case of a solitary lipoma, there may be an indication for liposuction if the diameter is particularly large $(>10 \mathrm{~cm})$; smaller isolated lipomas can be removed faster and more effectively using a small excision $[7,10]$.

Axillary hyperhidrosis: It is a form of focal, pathologically increased sweating without cause. This idiopathic disorder, which can also affect other regions such as the palms or soles of the feet, is probably due to vegetative regulatory disorders. These are very unpleasant for the sufferer, and difficult to control with therapy. At the end of the 1980s, axillary liposuction for the treatment of hyperhidrosis was first described. It is now an established therapeutic approach. Even if this procedure cannot remove all of the sweat glands, in many cases it is possible to achieve a normalization of sweating. Recurrence is due to regrowth of the glands from the remains of secretion tubes, and must be distinguished from what is known as "pseudo-recurrence", in which patients doubt the success of treatment when they still sweat to a physiological extent after the procedure $[7,10]$.

Skin flap mobilization: Local flap-plasty is often used to conceal larger skin defects, e.g. following tumor resection. This requires mobilization of the skin for the intended flap, which is normally done using scissors. The use of blunt liposuction cannulas has proved to be a minimally traumatizing, fast, and effective procedure for the mobilization of large skin flaps. It may also be possible by removing small quantities of fat from the flap by aspiration to reduce the tension when closing the skin $[7,10]$.

Other indications actually described are obesity and lower limb arthritis, multiple symmetric lipomatosis (Madelung disease or Launois-Bensaude syndrome), Melkersson-Rosenthal syndrome, Dercum disease, limb gigantis and macrodactyly, scar revision, extraction of permanent fillers, extravasation injuries, genital area and sexual dysfunction [7]. Although some of the described indications of the various liposuction technologies are still not completely validated and standardized, they undoubtedly open new horizons for liposuction far from its original aesthetic indication. 
Page 3 of 11

\section{Devices and Modalities}

Traditional liposuction, as popularized by Illouz, is referred to as suction-assisted liposuction. Other common modalities include power-assisted liposuction, vibro-assisted liposuction, ultrasoundassisted liposuction, vaser-assisted liposuction, and laser-assisted liposuction [6].

Suction-assisted liposuction (SAL) remains the most common modality for liposuction. As stated above, it is considered "traditional liposuction," in which variable-size cannulas are employed, with an external source of suction for removal of aspirate. Although traditional liposuction is advantageous for large areas of fat removal or for multiple sites, manual syringe liposuction is well-suited for fine contouring areas such as the face and neck. Low negative pressure suction is created manually by pulling back on the syringe plunger. Various locking devices can be used to secure the plunger in a set position, creating a vacuum for suctioning. Manual syringe liposuction is most practical for harvesting smaller volumes of fat for autologous fat transfer. Studies have shown aspirant from power suction may have similar adipocyte viability in comparison to low pressure manual suction. However, because of the extensive fragmentation of lipocytes in ultra- sound-, power-, and laser assisted devices, aspirate from these newer technologies are not suitable for fat transfer $[6,10]$.

Power-assisted liposuction (PAL) was first introduced by Charles Gross, an American surgeon; it involves an external power source driving the cannula, which is variable in size and flexible, in a $2 \mathrm{~mm}$ reciprocating motion at rates of 4000 cycles per minute. The mechanism action is due to a jackhammer-type movement of the cannula tip which breaks up fat, and the fat aspirated into the cannula openings is avulsed by the reciprocating motion.

Advocates of PAL contend that it is best used for large volumes, fibrous areas, and revision liposuction. Compared with other devices is similar in terms of complications, speed of recovery, and aesthetic results, but is superior in terms of ease of fat removal. Previously, its utility was limited because of noise generation from an external power source (gas or compressed air) and the mechanical vibration experienced by the operating surgeon. PAL modalities now run on electrical power sources; hence, vibration and noise have decreased significantly. PAL is said to be a handy technique, with the most favourable cost-benefit ratio, and seems to be the best option for busy liposuction practices or fast office procedures $[6,10]$.

Vibro-assisted liposuction (VL) represents a development of the PAL concept. In this system, the cannula is activated by air pressure, producing a complex movement of the tip. This movement, combining antero-posterior, supero-inferior and parasaggital displacement is called "nutation". The amplitude of this movement depends on the cannula length and diameter as well as the pressure entering the handpiece. A recent publication by Rebelo describes this technique and a study conducted by O. Heymans et al, showed that vibroliposuction is more efficient than SAL. It removed $40 \%$ more fat than SAL under the same conditions, complications were even fewer than with the previous use of SAL. They conclude this technique is less traumatic because fat extraction is more efficient needing fewer passes of the cannula. VL respects the lymphatic vessels and neurovascular bundles.

The combination of VL and open procedures showed the neurovascular structures to have been left intact, in the meshed tissue, and hematomas were fewer, compared with the previous combination with SAL $[6,10]$.
Ultrasound-assisted liposuction (UAL) was developed by Zocchi in the 1980s to improve the penetration through fat including fibrous areas while decreasing the work of the surgeon. UAL utilizes ultrasound energy to break down fat and allow removal. Its mechanism of action is primarily mechanical in nature, but cavitation and even thermal effects may occur. With this technique, fat is emulsified, creating subcutaneous microcavitations before aspiration, which allows removal through traditional liposuction cannulas. UAL is designed to work in conjunction with traditional liposuction as a pretreatment method for difficult to treat areas before suction lipoplasty. Advantages include less surgeon fatigue, improved results in fibrous areas and in secondary procedures, less bloody aspirant and may reduce local trauma, decreasing postoperative ecchymoses and edema in patients. Disadvantages have been reported to include larger incisions, longer operative times, and the possibility of thermal injury. Controversy exists whether UAL improves esthetic outcome and enhances skin retraction. UAL requires a superwet environment and cannot be performed without a wetting solution. Also, appropriate management of the UAL probe is essential to preventing thermal injury to the superficial dermis; hence, skin protectors are often employed $[6,10]$. Vaser-assisted liposuction (VAL) employs a newer generation ultrasound-assisted liposuction device that incorporates less energy with more efficient, solid probes. The probes come in an array of sizes and grooving, depending on tissues in which they will be used.

Only small-diameter solid probes $(2.9$ and $3.7 \mathrm{~mm}$ ) are used and require much less ultrasound energy than the traditional UAL systems currently used, decreasing its thermal component to the tissues. Grooves near the tip are added to increase fragmentation efficacy. The VASSER still liquefies fat, but the risk of thermal injury (from end blows and at the insertion site) is reduced. In many ways, this new technology is more like power-assisted lipoplasty than traditional internal ultrasound-assisted lipoplasty. However, skin protection (ports and wet towels) is still needed. Garcia and Nathan showed the VAL to have significant advantages in blood loss and recommended its use in large-volume liposuction procedures and areas of fibrous fatty tissue where excess blood loss is expected $[6,10]$.

Laser-assisted liposuction (LAL) has been at the forefront of marketing hype for the past several years. The treatment involves insertion of a laser fiber via a small skin incision. Depending on the manufacturer, the fiber may either be housed within a cannula or stand alone. The negative suction draws the fat globule into the hole of the cannula where the laser beam (YAG laser $40 \mathrm{~W}$ ) shears it. There are several commercially available lasers on the market under different trade names. Most companies and physicians utilizing this technique employ a four-stage technique: infiltration, application of energy to the subcutaneous tissues, evacuation, and subdermal skin stimulation. The laser fiber acts to disrupt fat cell membranes and emulsify fat. Evacuation then commences via traditional liposuction cannulas. The release of fat by suction is facilitated, surgical trauma is diminished, ecchymosis or hematoma is reduced and patient recovery is fastened. The main disadvantages are the slightly cumbersome and awkward equipment, and the fact that experience in laser use is essential. Safety glasses are necessary, the procedure is noisy and constant cooling is required.

In 2004, Brown et al. analyzed the effect of low-level laser therapy on abdominal adipocytes before lipoplasty procedures and their results did not bear out the effect of low-level laser therapy on adipocyte structure; the only advantages found were greater ease and less arm 
motion fatigue. A previously published randomized, doubleblinded controlled study by Prado et al., showed no difference in the outcomes of LAL versus traditional SAL. In that study, each patient served as his or her own control. Factors evaluated included cosmetic result, postoperative pain, length of operation, lipocrit, and free fatty acids. Besides the lack of difference in the cosmetic outcome of LAL versus SAL, the authors also reported a longer operative time with LAL, less early postoperative pain with LAL, and elevated free fatty acids/ triglycerides in the laser-treated lipoaspirate $[6,10]$.

\section{Anesthesia}

The choice of anesthesia technique for liposuction varies based on multiple factors: surgeon and anesthesiologist preferences, patient comorbidities, patient positioning, anatomical areas being treated, type of liposuction being performed, amount of expected lipoaspirate, length and extent of procedure and volume of liposuction planned [9].

Descriptions of local anesthesia, various forms of sedation (mild, moderate, heavy) and general anesthesia are present in the literature. Small-volume liposuction cases can be performed with local anesthesia, with or without mild sedation. Complex, large-volume liposuction and combined cases should be performed under general anesthesia. Deep sedation cases and general anesthesia procedures are performed under supervision of board-certified anesthesiologists in licensed surgery centers or hospitals [11].

The monitored anesthesia care is a useful technique for small volume liposuction. Infiltration with vasoconstrictor and local anesthetic reduces bleeding and provides intraoperative analgesia, respectively. Thus, it allows liposuction to be performed under light sedation, implying a short recovery time, earlier discharge, and low cost to the patient. However, if infiltration is not uniform, some areas will have a lack of analgesia, thus requiring more sedation [12].

Sedation with midazolam (1-3 mg) and analgesia with fentanyl (25-50 mg) or remifentanil (12.5-25 mg) is commonly employed for small volume liposuction. Propofol $(0.5-1 \mathrm{mg} / \mathrm{kg})$ may be given intermittently for monitored anaesthesia care. Ketamine in low doses $(0.25-0.5 \mathrm{mg} / \mathrm{kg})$ along with midazolam decreases significantly the consumption of opioids in the intraoperative period and of analgesics in the postoperative period. Clonidine $2-5 \mathrm{mg} / \mathrm{kg}$, is also a useful adjuvant in sedation techniques [12].

The lumbar epidural anesthesia is widely used to provide analgesia in abdominal liposuction. The quality of analgesia provided is superior to that provided by local infiltration. Epidural anesthesia is associated with a lot of minor side effects, easy to prevent and to manage and that commonly do not raise safety concerns. However, an extensive epidural blockade is frequently associated with hypotension and must be limited to patients with good cardiac reserve [13].

Spinal anaesthesia (also called subarachnoid block) is preferred as bulk of fat to be sucked out is situated in lower half of the torso (below the umbilical area) and the duration of surgery is about three hours. This covers the subcostal areas as well and further reduces the need of lidocaine in the infiltrating solution as general anaesthesia is avoided. A 27 gauge needle avoids the often troublesome post spinal anaesthesia headaches. Fentanyl is additionally added to the high spinal because it has a bupivacaine sparing effect on spinal anaesthesia.

The quality of anaesthesia and muscular relaxation is excellent and administration of opioids in the subarachnoid space provides good analgesia in the first 24 hours of the postoperative period. In these conditions, this technique is safe, low cost, and with few side effects $[12,14]$.

There are two reasons why infiltration with local anesthesia has traditionally been limited to relatively small areas of skin: (1) the stinging pain associated with infiltrating the local anesthesia is not easily tolerated, and (2) published dosage limitations have precluded anesthetizing large areas of skin. These limitations have now been overcome with the recognition that (1) adding sodium bicarbonate in order to neutralize the acidity of commercially available local anesthesia solutions of lidocaine and epinephrine dramatically reduces the usual burning-stinging pain of infiltration, and (2) using dilute solutions of lidocaine with the tumescent technique permits profound anesthesia of very large areas [15].

The tumescent technique (detailed later) involves the subcutaneous infiltration of very large volumes of dilute local anaesthetic solution (usually containing adrenaline), until the tissues become tense and swollen. This technique permits regional local anesthesia of skin and subcutaneous tissue by direct infiltration rather than by proximal nerve block or even general anesthesia, without IV sedation or narcotic analgesia [15]. The tumescent anesthesia associated with unconscious sedation, has been demonstrated to be a safe technique, without major complications, with lower operative time, quick recovery and good satisfaction in a large number of outpatients. Decreased operative time moreover reduces risk of thromboembolic phenomenon, heat loss, and the anaesthetic solution exposure time, with lower absorption risk. However, this despite controversial data reported in literature [16].

Data from the few anesthesia studies that have specifically assessed patients undergoing liposuction confirm the safety of general anesthesia, epidural anesthesia, spinal anesthesia, moderate sedation, and local anesthesia for this procedure. It should be noted, however, that epidural anesthesia and spinal anesthesia can cause vasodilation and hypotension, thereby necessitating the administration of excess fluid and increasing the risk for fluid overload [5]. Although no evidence supports the use of any single technique, the American Society of Plastic Surgeons Practice Advisory recommends avoiding neuraxial anesthesia (i.e., spinal, epidural) in office-based settings because of potential hypotension and volume overload issues; therefore its use must be reserved for the operation room. Also, a moderate sedation/analgesia (intravenous or oral) augment the patient's comfort level and is an effective adjunct to anesthetic infiltrate solutions [17].

\section{Infiltration Solutions}

The terminology relating to infiltration of the subcutaneous fat before liposuction includes: the dry, the wet, the superwet and the tumescent technique [18]. The dry and wet techniques are now of historical interest only. In the dry technique, liposuction was performed without the addition of subcutaneous solution injection. The wet technique was introduced by Illouz in 1984 and consisted of injecting hypotonic saline solution inducing hydrolysis of fat cells (lypolysis). There remains no clinical evidence to support this action mechanism and the use of hypotonic saline has fallen into disfavour. The use of saline infiltration, however, gained popularity, and by the early 1980s the majority of surgeons were using the wet technique. They infiltrated 200 to $300 \mathrm{cc}$ of saline, with or without additives (lidocaine and adrenaline) into a surgical area7. Much attention was paid to wetting solutions in the 90's, particularly maximum and safe 
Page 5 of 11

doses of lidocaine and appropriate replacement fluid volumes achieved by intravenous and oral administration and by means of hypodermoclysis. These two techniques were both abandoned because of excessive blood loss; the suction aspirate containing $20-45 \%$ of blood in the dry technique and $4-30 \%$ in the wet technique [9].

New solutions appeared with the superwet technique and the tumescent technique, which dramatically improved the safety of liposuction. The superwet technique is defined as $1 \mathrm{ml}$ of infiltrate per $1 \mathrm{ml}$ of aspirate. The tumescent technique is defined as $2-3 \mathrm{ml}$ of infiltrate per $1 \mathrm{ml}$ of aspirate. In these methods, the subcutaneous fat is infiltrated with large volumes of a mixture of lidocaine, adrenaline, sodium bicarbonate and normal saline before fat removal.

Blood loss dropped to 1 percent of the aspirate, which allows a larger volume of fat to be safely aspirated. Another advantage is the reduced need for intravenous administration of fluids perioperatively [19].

Recently, little has changed with regard to the best evidence for lidocaine and epinephrine concentrations in wetting solutions. Lidocaine use continues to vary between surgeons; however, some studies have demonstrated that the lidocaine component of wetting solutions can be eliminated without increased postoperative pain $[9,20]$.

Despite the relatively low concentrations infiltrated, the use of lidocaine and epinephrine may result in toxicity in some cases. Lidocaine toxicity has central nervous system and cardiac effects, with the first signs of toxicity being circumoral numbness, tinnitus, and lightheadedness. Intraoperative manifestations may include arrhythmias. Increasing levels cause tremors, seizures, and eventually cardiac and respiratory arrest [21].

For the majority who do use lidocaine, published reports demonstrate lidocaine use up to $55 \mathrm{mg} / \mathrm{kg}$ without complications, although most surgeons seem to prefer far lower doses that still achieve satisfactory analgesia alone or with systemic anesthesia $[20,21]$. Klein's data showed that levels of $35 \mathrm{mg} / \mathrm{kg}$ were safe in liposuction [18].

Epinephrine induces vasoconstriction, improving haemostasis, delays absorption of the anaesthetic agent, prolongs its effect to four times as long, decreases the amount needed and reduces the risk of lidocaine toxicity. Epinephrine has been shown to have increased cardiovascular effects after infiltration and peaks at five hours after infiltration. Caution is warranted in patients with diminished cardiac reserve and/or questionable heart history; it is recommended that 7 $\mathrm{mg} / \mathrm{kg}$ not be exceeded, although doses as high as $10 \mathrm{mg} / \mathrm{kg}$ already have been used safely $[9,21]$.

Other studies recommend the use of bupivacaine, or assess lidocaine and bupivacaine concentrations in superwet fluids and serially measure their levels postoperatively, and firmly advocate for their necessity in pain control. These studies report good results with the use of bupivacaine and did not report any episodes of toxicity. The only episodes of toxicity reported in the survey were associated with lidocaine use [21].

There is still no consensus regarding the optimal composition and amount of subcutaneous infiltration solution for safety or for optimal aesthetic results. With these techniques, the focus has shifted from hypovolemia prevention to the prevention of fluid overload. The risk of fluid overload and congestive heart failure seems to be lower with the superwet technique $[9,22]$.

\section{Fluid Resuscitation}

Body contouring procedures can result in significant fluid shifts and intravascular volume changes for the patient. The operating surgeon should maintain a dialogue with the anesthesia provider, so that patients receive adequate replacement volume and proper fluid resuscitation. Often, a Foley catheter is utilized to guide intraoperative and postoperative volume resuscitation. Awareness of four key elements will guide the intraoperative fluid management of liposuction patients: intravenous fluid maintenance (body weight dependent), third space losses, volume of wetting solution infiltrated, and total lipoaspirate volume [1].

Large-volume liposuction patients can present an especially difficult challenge for fluid resuscitation. As previously mentioned by Rohrich et al in 1998 (updated in 2006), the following formula aids in fluid management for these patients, nevertheless, it serves as a guideline and is not meant to replace sound clinical judgment based on specific patient needs [23]:

\section{Replace losses from preoperative oral intake loss as needed.}

2. Maintain fluid throughout the procedure and manage it based on vital signs and urine output.

3. Employ the superwet infiltration technique

4. Administer crystalloid replacements, $0.25 \mathrm{ml}$ for each $\mathrm{ml}$ of lipoaspirate over $5 \mathrm{~L}$.

Profound metabolic alterations accompany large volume liposuction. Because of the increasingly large volume of infiltrate used in larger volume liposuction, careful attention must be paid to all fluid infused and whether it is part of the infiltrate solution or part of IV fluids administered during the procedure [17].

Accurate intake and output monitoring of all fluids utilized in the operative and postoperative periods must be made, communication with the anesthesia care provider on fluid management is critical, fluid management in liposuction surgery must account for maintenance requirements, preexisting deficits and intraoperative losses of aspirated tissue and third space deficits, preexisting fluid deficits should be minimal after an overnight fast, blood loss estimates should be made and confirmed with pre- and postoperative hemoglobin measurements (however, due to fluid shifts, hemoglobin levels may not be reliable during the first 24 hours postoperative period), and calculation of residual fluid volumes after liposuction is helpful in planning postoperative care [17].

\section{Intraoperative Care}

There are several precautions that can be taken intraoperatively to maximize the postoperative recovery [17]:

1. Preserve body core temperature with approved warming devices.

2. Position patients properly on padded operating table with knees slightly flexed so as to maximize blood flow through the popliteal vein.

3. Intermittent pneumatic compression devices should be used intraoperatively to prevent deep vein thrombosis, particularly with patients at moderate to high risk of blood clots. Low molecular weight heparin may also be administered to those patients at higher risk. 
Page 6 of 11

\section{Postoperative Care}

Immediate postoperative care should include an assessment of fluid and electrolyte balance and the administration of replacement fluids, as needed. In addition, red blood cell loss needs to be assessed and replacement transfusions should be given, if needed. Patients who undergo large-volume liposuction or multiple procedures should be warmed during recovery using appropriate warming methods $[5,17]$.

At the conclusion of surgery, patients are placed in the appropriate compression garment; some type of compression foam may be used. Compression garments are worn at all times for the first 2 weeks followed by nightly for an additional 2 weeks [17,22].

Depending on the volume of lipoaspirate and other concomitant procedures, patients are either discharged home or remain overnight in a surgical hotel suite/hospital. In general, patients who have undergone any large-volume procedure (more than $5000 \mathrm{ml}$ aspirate), liposuction of multiple areas, or liposuction in addition to abdominoplasty are kept overnight for 24-hour observation [22].

Patients are asked to ambulate the night of the surgery, and sequential compression devices remain on the patient while in bed until discharge. Before the patient is discharged, he/she must be alert and oriented with stable vital signs. The patient should expect significant bruising and swelling for at least the first 48-72 hours $[17,22]$.

Patients are allowed to shower beginning one or two days postoperatively and instructed to keep the compression garment on 24 hours a day for 2 weeks, except while showering $[1,22]$. The majority of small-volume patients can be counseled that they may return to work in 3-5 days, whereas, generally, 7-10 days is needed for patients undergoing larger volume procedures. Most patients will return to regular activities in 3 to 4 weeks [22].

Patients are cautioned that swelling may persist for 3 to 4 weeks and that final results can be expected in 3 to 4 months. Postoperative lymphatic massage is encouraged to help with swelling and induration. This is often begun prior to surgery and resumed shortly after the procedure [22].

Correction of deformities and/or revisions should generally be undertaken at least 3 to 6 months after the original liposuction procedure to allow for tissue normalization. Deformities may be corrected with repeat liposuction and/or fat grafts [5].

\section{Safety Guidelines}

More severe consequences of lipectomy include not only morbidity but also mortality. In a review by Grazer and de Jong of 1200 active board-certified plastic surgeons, there were 95 fatalities in nearly 500,000 lipectomy procedures, producing a mortality rate of 19 in 100,000 with $23 \%$ attributable to pulmonary embolism 22 . In a discussion of these findings, Rohrich and Muzaffar suggested the following Safety Guidelines in Liposuction [24]:

1. Appropriate patient selection (American Society of Anesthesiologists class I, within $30 \%$ of ideal body weight).

2. Use of superwet technique.

3. Meticulous monitoring of volume status (urinary catheterization, noninvasive hemodynamic monitoring, constant communication with anesthesiologist).

4. Judicious fluid resuscitation.

5. For aspirate $<5$ L: maintenance of fluid plus subcutaneous infiltrate.

6. For aspirate $>5 \mathrm{~L}$ : maintenance of fluid plus subcutaneous infiltrate plus $0.25 \mathrm{ml}$ on intravenous crystalloid per $1 \mathrm{~mL}$ of aspirate $>5 \mathrm{~L}$.

7. Overnight monitoring of large-volume ( $>5 \mathrm{~L}$ total aspirate) liposuction patients in an appropriate healthcare facility.

8. Use of pneumatic compression devices in cases performed under general anesthesia or lasting longer than 1 hour.

9. Maintaining total lidocaine doses below $35 \mathrm{mg} / \mathrm{kg}$ (wetting solution).

There is no scientific data available that support a specific volume maximum at which point liposuction is no longer safe, although the risk of complications is unavoidably higher as the volume of aspirate and the number of anatomic sites treated increases. It is important to note the distinction between total fat removed and total aspirate removed. Total aspirate is defined as the combination of total fat and fluid removed during liposuction. Total aspirate should be the method used when tracking the volume of liposuction removed [17].

Regardless of the anesthetic route, large volume liposuction (greater than 5,000 cc total aspirate) should be performed in an acute-care hospital or in a facility that is either accredited or licensed. Postoperative vital signs and urinary output should be monitored overnight in an appropriate facility by qualified and competent staff who are familiar with perioperative care of the liposuction patient [17].

\section{Complications}

Through the years, the majority of the world medical literature has shown that lipoaspiration is a safe surgical procedure. Recently, reports such as those from Rao et al. have emerged with alarming information about deaths accompanying lipoaspiration25; those reports have generated diverse reactions and controversy.

According to Grazer and de Jong the mortality rate for liposuction is 20 per 100,000 , similar to the one associated with traffic road accidents in the United States [26]. They have identified a total number of 130 deaths in almost 500,000 surgical procedures, of which the most important causes were thromboembolism (23.1\%), abdomen or viscera perforation (14.6\%), anesthesia/sedation/medication (10\%), fat embolism (8.5\%) and others (43.9\%). Most reported cases in which liposuction had a lethal outcome were done using the tumescent liposuction [26]; laser assisted lipoaspiration being known to led less frequently to severe complications.

Complications can be systemic or local, can occur in the perioperative period, early postoperative period and late postoperative period and have been categorized as major or minor. Mayor complications include deep venous thrombosis and pulmonary embolism, hypothermia, lidocaine and epinephrine toxicity, cardiopulmonary arrest and fluid shifts, sepsis, fat emboli, perforation of abdomen and viscera. Minor complications include hematoma, seroma, surface irregularities, skin excess, cutaneous hyperpigmentation, skin necrosis, cutis marmorata $[25,26]$. 
Deep venous thrombosis is one of the most feared complications due to its relation to pulmonary embolism, which has been the main cause of death among patients undergoing cosmetic surgery claiming one fourth of the deaths [25]. Incidence of deep venous thombosis in liposuction has been reported at $<1 \%$, but a marked increase in this percentage is demonstrated when liposuction is combined with other surgery (abdominoplasty/belt lipectomy) [15]. Clinical signs of lower extremity swelling, Homans sign, shortness of breath, chest pain, tachycardia, coughing up blood, sweating and anxiety should alert the provider to the possibility of deep venous thrombosis/pulmonary embolism and warrant immediate evaluation and treatment [26,27].

The use of preventive measures (stockings, pneumatic intermittent compression systems, etc.) together with early mobilization, appropriate hydration and anticoagulation when indicated are sufficient to prevent venous thrombosis in healthy individuals. Administration of enoxaparin has resulted in a decreased incidence of deep venous thrombosis but there may be increased risks of bleeding. Chemoprophylaxis is used at the discretion of the surgeon, based on risk assessment evaluation [9].

Hypothermia occurs commonly in liposuction cases. Hypothermia is generally defined as a core body temperature less than $36.5^{\circ} \mathrm{C}$. It has influence not only in the coagulation system but also affects the immune system as well. Its risk is amplified in larger volume cases where more of the patient is exposed at one time. Because of this proper precautions have to be taken to prevent excessive heat loss. The use of warming devices, warm fluids and attention to room temperature are the basic steps to prevent hypothermia [27].

Fluid management is crucial to the prevention of volume overload and anesthesia related complications. The tumescent liposuction technique has been implicated in volume overload and pulmonary edema; however, these can often be attributed to incorrect patient selection and/or poor fluid management. Proper preoperative, intraoperative and postoperative fluid management is essential to optimize the good perfusion and minimize the risk of cardiopulmonary complications and death. Fluid aspirations should be limited to $5 \mathrm{~L}$ per session to avoid the excessive third spacing that could jeopardized the ability to compensate the fluid shifts on the average patient. Other rules are to limit the aspirate to less than $5 \%$ of the body weight and treat less than $30 \%$ of the body surface [28].

Although of rare occurrence, fat emboli could lead to fatal outcomes. Fat embolism syndrome (further detailed later) presents with a triad of petechial rash, respiratory distress and cerebral dysfunction. The diagnosis is difficult and the treatment is supportive. Corticosteroids may play a role in the management of this rare entity [26,29].

Wound infections and necrotizing soft tissue infections are rare but can occur after liposuction. Erythema, drainage or even swelling should not be taken lightly. Unrecognized or untreated infections could lead to compromise of a large surface area or even to necrotizing fasciitis and other more severe systemic manifestations. Local sings of infection (persistent postoperative fever and/or cellulitis) should be closely monitored and aggressively treated. First-generation cephalosporins are administered perioperatively prior to incision unless the patient has a known history of methicillin-resistant Staphylococcus aureus in which case vancomycin is administered preoperatively.

Hematoma may result from inappropriate technique or increasebleeding diathesis from congenital vs. acquired reasons. The use of wet, superwet or tumescent liposuction has decreased significantly the risk of bleeding after suction lipectomy [29].

Seromas are related to an excessive liposuction with inappropriate postoperative management. Seromas are rare and thought to be secondary to overzealous treatment of an area, which denudes the fascia. It appears to be technique dependent rather than technology specific. A loose closure of cannula sites, postoperative compression garments (may provide comfort and at the same time decrease the death space suitable for fluid accumulation) and expressing residual fluid over liposuction areas at the end of procedure all reduce the incidence of seroma formation and edema. Some advocate the use of drains over the first 24 hours in cases of large volume liposuction as well as manual drainage $[1,25,29]$.

Postoperative edema and ecchymosis occur to a varying extent in all patients. Prolonged edema can occur up to three months from surgery and is best treated with supportive care and lymphatic massage. Postoperative paresthesia/dysesthesia can occur in all forms of liposuction. The sensory changes are usually reversible and can take up to 10 weeks to recover [29].

\section{Fat Embolism Syndrome}

A literature review yielded only a few case reports of fat embolism syndrome (FES) after liposuction; however, this does not underscore its importance as it is a fatal syndrome that is difficult to diagnose. The clinician must be astute to this potential consequence as it can share features with the more common complications of liposuction, notably pulmonary embolism and sepsis secondary to necrotizing soft tissue infection [30,31].

Fat embolism syndrome occurs in $5-10 \%$ of the cases, but are usually minor, consisting primarily of anesthesia, seroma, edema, pigmentation, pain, and hematoma. The overall mortality from FES after liposuction is approximately $10-15 \%$ with higher mortality associated with fulminant FES due to severe right heart failure compared with FES in which the mortality relates largely to underlying respiratory failure (or rarely cerebral edema causing brain death) [32].

The relationship between FES and liposuction has been established in the animal model. In their study, El-Ali and Gourlay (reference) found lipid deposits in the lungs of every laboratory rat that underwent 30 minutes of liposuction. Kenkel et al. (reference) found similar results in their porcine model. The pathogenesis of FES after liposuction involves both mechanical and biochemical means. Direct inoculation of fat globules into the venous circulation after mechanical rupture of these vessels can directly inhibit blood flow through pulmonary capillary beds. The pulmonary arterial pressure rises and can push the emboli into the systemic circulation where it can cause end-organ damage [33].

In clinical practice, saline solution (with or without lidocaine or epinephrine) is usually used to infiltrate the subcutaneous fat before liposuction. This fluid is meant to expand the target tissue and facilitate the passage of the cannula, and thus make liposuction more safe and efficient. It has also been found to reduce blood loss as a percentage of the aspirate and, as such, increase the amount of lipid that can be aspirated safely [32].

Several authors have indicated that it seems likely that there is an incidence of subclinical fat embolism after liposuction, and that the clinical picture can vary from subclinical hypoxia to full-blown adult respiratory distress syndrome, which can lead to coma and death [32]. 
Page 8 of 11

The three classic symptoms of FES, respiratory distress, cerebral dysfunction, and petechial rash, usually appear within 24-72 $\mathrm{h}$ after the procedure. Other symptoms of fat embolism include tachycardia, tachypnea, fever, hypoxemia hypocalculium, hypocapnia, thrombocytopenia, and occasionally mild neurologic symptoms. Arterial blood gas analysis is important because the development of clinical syndromes can cause $\mathrm{PaO} 2$ to drop to $50 \mathrm{mmHg}$ or lower $[33,34]$.

Imaging findings play an important role in confirming the diagnosis of FES. Chest radiography, noncontrast CT scan, highresolution CT, ventilation-perfusion imaging, MRI, transcranial Doppler sonography, and intraoperative transesophageal echocardiography are included [33].

Fat embolism syndrome is a self-limiting disease and symptoms usually resolve within 1 week; therefore the treatment of FES is mainly supportive, aimed at improving respiratory condition and hemodynamic stability [35]. The use of high-dose corticosteroids may limit the increase in circulating free fatty acids and reduce systemic inflammation, but its use is controversial and the dose and optimal timing of administration have not been established and should be further studied to make certain. Low-molecular-weight dextran is helpful for decreased blood viscosity, reducing platelet adhesion, reversing thrombocytopenia, and reducing cell aggregation. Nitric oxide and prostacyclin are considered beneficial in pulmonary supportive therapy $[33,35]$.

The main objectives to reduce the presence of this rare but with high mortality disease are the following: careful selection of patients and techniques, reduction of surgical time and the amount of fat aspirated, limiting the number of procedures in one sitting, careful attendance and patient postoperative monitoring with intravenous fluid administration for up to 24 hours postoperatively and not release the patient from the hospital the day of the procedure. Finally, if FES is present, we should make an appropriate and aggressive attendance, because prognosis will be more favorable, reducing the morbidity and mortality [33].

Although in skilled hands, liposuction is an effective and safe technique for body contouring, it is not without risk. Fat embolism syndrome may occur and may not be as uncommon as previously thought. It is extremely important to minimize the risk of complications with preventive strategies and appropriate management [36].

\section{Outcomes}

Follow-up studies, although limited in quantity, have sought to characterize the long-term results of patients undergoing liposuction. In work derived from a survey distributed to 600 liposuction patients, several key findings were discovered. Notably, a responder's opinion of their appearance was the pivotal determinant in their satisfaction with their liposuction procedure. This, in turn, influenced whether they would have the procedure again or recommend it to another [22].

Other factors, including weight gain, revision rate, the return of fat, and the level and duration of postoperative pain, did not have a statistical effect on these decisions. However, weight gain has been shown to have a direct negative impact on appearance, which, in part, impacts their satisfaction, willingness to continue therapy, and, ultimately, outcomes [22].
These findings reiterate what has already been emphasized in body contouring, proper patient selection and physician-patient dialogues are crucial given that those patients who were not satisfied with their liposuction treatment were also those with the lowest opinion of their appearance. In a separate publication reviewing the same data, the authors relate the importance of educating patients on postoperative lifestyle changes, including continued exercise and healthy eating, which are paramount to successful liposuction treatment $[9,22]$.

\section{Metabolic Changes after Liposuction}

Metabolic syndrome is epidemiologically important medical condition that includes insulin resistance, dyslipidemia, central obesity, hypertension, impaired glucose tolerance or diabetes mellitus, and high rates of atherosclerotic disease [37]. Adipose tissue was for a long time regarded as a relatively passive site of energy storage. Recent studies indicate that adipose tissue is an endocrine organ producing numerous proteins, collectively referred to as adipokines, with broad biological activity, which play an important autocrine role in physiology and are involved in obesity-associated complications. Adiposity contributes to a pro-inflammatory milieu, playing an important role in mediating all phases of atherogenesis, leading to the development of atherosclerosis and metabolic outcomes [38].

The importance of body fat distribution and its effect over insulin sensitivity initially was evaluated on transversal studies as well as in cohort studies. In these studies, it was established that abdominal fat is a factor that greatly influences the development of insulin resistance more so than peripheral fat, also known as femoral-gluteal fat. Likewise, major interest exists in abdominal fat with its visceral component and its relationship with metabolic syndromes and cardiovascular morbidity-mortality. During the first observations of the influence of abdominal fat components, either visceral or subcutaneous, relating to insulin resistance, it was found that visceral fat was more important in this regard. Nevertheless, with newer measurement techniques of the distribution of fat, such as nuclear magnetic resonance and computed tomography, the association of visceral fat uniquely linked with the metabolic syndrome is controversial [39].

Because of this, some conducted studies conclude that subcutaneous abdominal fat is an important predictor, independent of visceral fat, in insulin resistance syndrome. Zamboni et al found that weight reduction in premenopausal women resulted in a greater loss of visceral fat than subcutaneous fat, with resulting improvement in lipid concentrations and an increase in insulin sensitivity. However, visceral fat is a smaller component compared with subcutaneous fat, giving primary importance to the latter [39].

Although large-volume liposuction of subcutaneous fat only slightly alters the total amount of fat tissue, it has been considered that after liposuction more subcutaneous fat than visceral fat is lost. This produces a preponderance of visceral to subcutaneous fat, According to the latest findings, which changes the abdominal-superficial adipose tissue ratio and thus might affect the potential of metabolic syndrome by the means of its separate parameters and clinical manifestations. Nonetheless, other authors have encountered a beneficial effect on insulin sensitivity following liposuction, especially those studies realized in obese women [37,40].

The first study to assess the impact of liposuction expressed in terms of changes in the absolute amounts and in the proportions of subcutaneous and visceral adipose tissue was made by Matarasso et al; 
they analyzed in 63 normal weight women and 12 men the relative changes in total body fat and in visceral adipose tissue (nonsubcutaneous tissue) after largevolume liposuction (>1000 cc). They found that one third of the women had a mean increase of $16 \%$ in the proportion of visceral fat and $14 \%$ increase in the $12 \mathrm{men}$. They concluded that, although large-volume subcutaneous liposuction removed relatively little body fat, it led to significant increases in the proportion of visceral adipose tissue. Because the proportion of visceral adipose tissue is a risk factor for metabolic complications of obesity, the metabolic effects of large-volume liposuction need to be evaluated [41].

In a recent clinical study, Lazaro Cardenas-Camarena et al., determined whether the removal of abdominal subcutaneous fat via liposuction in 15 healthy non-obese women would influence insulin sensitivity and secretion, including serum concentrations of cholesterol, and the effect that the modification of these percentages has on body mass and adiposity. Regarding the serum levels analyzed, they found that the serum concentration of glucose and cholesterol and the percentage of adipose tissue decreased after liposuction. On the other hand, they didn't found a significant modification in insulin sensitivity as compared with the insulin estimated by HOMA IR, nor to a significant degree in serum insulin concentrations, nor in weight after liposution. They conclude that liposuction is a metabolically safe procedure because the modification of markers, such as cholesterol, insulin secretion, glucose, and adiposity reduction, may retard the appearance of the metabolic syndrome. However, they affirm that new investigations are necessary to study long-term results of liposuction relating to the metabolic profile [39].

Swanson evaluated the effect of liposuction and abdominoplasty on lipid levels and complete blood count, founding highly significant $(p<0.001)$ reductions in triglyceride level in patients with elevated preoperative levels and a significant decrease in white blood cell count $(\mathrm{p}<0.001)$ which are favorable metabolic effects of liposuction and liposuction/abdominoplasty, and are likely caused by a reduction in subcutaneous fat volume. Cholesterol levels were unaffected [42].

Only few studies have specifically investigated the metabolic sequelae of liposuction in obese women, as liposuction is currently reserved for most to treatment of focal areas of lipodystrophy that are resistant to conventional means of improvement such as diet and exercise. Moreover, a retrospective clinical study casts some doubt on the metabolic safety of large-volume liposuction by hypothesizing an alteration of the proportion of subcutaneous to visceral adipose tissue and thus a paradoxical increase of insulin resistance and associated sequelae [42].

Gonzalez-Ortiz el al evaluated the effect of surgically removing subcutaneous fat on the metabolic profile and insulin sensitivity in 12 young obese women after large-volume liposuction treatment. One month after liposuction, a significant decrease of fasting glucose was observed, associated with an improvement of insulin sensitivity (25\%) $(\mathrm{p}<0.045)$ assessed by the insulin tolerance test [40].

Results that can be found in the published literature remain controversial and often contradictory, thus leaving enough space for further investigations regarding the relation of these two clinical entities. It has been suggested that the plastic surgeons may play a significant role in body contouring of the obese patient at the end of the lengthy process of bariatric surgery and massive weight loss, most of all because fat removal produces beneficial effects on insulin resistance and vascular inflammation in the obese patient, reducing cardiovascular risk $[39,40]$.

Liposuction is usually recommended to treat body disproportion, not obesity. However, recent advances in liposuction techniques now make it possible to remove considerable amounts of subcutaneous adipose tissue [42]. Thus, liposuction may be a potential treatment for the metabolic complications of obesity [40]. Also, many of the patients have used liposuction as a stepping stone to behavior modification. Therefore, citing textually Dr. Louis DeLuca:

"Liposuction on obese individuals should be important to any aesthetic surgeon who truly believes that the treatment goal is to provide both an aesthetic and behavioral benefit, rather than simply the former". In my personal opinion, I think we have to define what are the expected objectives of liposuction in each case, taking into consideration that the metabolic effects of liposuction may vary in patients with overweight or obesity compared with patients with a normal weight.

\section{Other Horizonts: Autologous Fat Transfer and Stem Cells}

Although peripherally related to liposuction, the topic of fat transfer is among the most current and still controversial topics in plastic surgery despite initial investigations going back more than 25 years.

According to important scientific advances such as refinement of fat grafting, discovery of adipocyte stem cells, and understanding an engraftment process of fat, interest about fat grafting cannot be higher than ever before and it is expanding the limit to new fields such as large volume fat grafting, fat transfer for burn and difficult wounds, and also for scar treatment [43].

Fat transfer may be performed as a primary procedure (e.g., breast or buttock augmentation), as an adjunct (e.g., face-lift surgery or breast reconstruction), or for the potential of "stem cell" therapy [9]. Numerous studies have looked at the ideal extraction method of fat, how it should be processed, how it should be injected, and the depth of injection. It is likely that no one method will apply to all autologous fat transfer procedures [7].

Fat grafting for breast augmentation has been already attempted since several decades ago, but complications of fat necrosis, cyst formation, and infections have long been big issues.

However, in these latter days, breast augmentation surgery using cell-assisted lipotransfer increases greatly and the fat grafting procedure in patients with partial mastectomy or lumpectomy to improve breast shape is already established as a stable treatment [43].

Many patients after breast reconstruction with flap operation or breast prostheses wants fat grafting to improve breast shape or to substitute breast prostheses for autologous fat. Lately, it is reported that application of the fat tissue to burn wound or extensive wound not only reduces therapy period but also reduces deformity after the end of healing process. Also, fat grafting is conducted to improve scar and scar contracture by injury or burn [43].

The American Society of Plastic Surgeons and the American Society for Aesthetic Plastic Surgery recently published a position paper on the topic of stem cell therapy to address the growing concern surrounding unsupported claims of stem cell treatments in aesthetic surgery [9]. They determined that terms such as "stem cell therapy" or "stem cell procedure" should be reserved to describe those treatments or 
Page 10 of 11

techniques where the collection, concentration, manipulation and therapeutic action of the stem cells is the primary goal, rather than a passive result, of the treatment. For example, standard fat grafting procedures that happen to transfer some stem cells that are naturally present within the tissue should be described as fat grafting procedures, not stem cell procedures.

Fat is a unique regenerative complex comprising adult stem cells as well as high energy resource related with tissue repair and regeneration. The regenerative potential of fat tissue led us to expand the limit of application day by day. Fat tissue is expected to be an essential component of regenerative medicine in the twenty first century and it is essential for plastic surgeons to understand new knowledge on fat biology to perform better and reasonable clinical practice [18]. However, the marketing of stem cell procedures as advantageous in aesthetic surgery is not adequately supported by clinical evidence at this time, and that all use of stem cell therapy in aesthetic and reconstructive surgery should be conducted within clinical studies under institutional review board approval [9].

\section{Conclusions}

Liposuction is one of the most common surgically performed procedures and has proven to be a safe, effective, and popular intervention for the surgical removal of adipose tissue. Its low complication rate supports the procedure's popularity, therefore should be considered a safe surgical procedure.

Over the past 30 years, there have been many advances in surgical technique and patient related standards for liposuction, resulting in improved safety and outcomes. The surgeon can now choose between a variety of liposuction techniques, cannula designs, and anesthesia options. New technology is continually being introduced, but we have to base treatment on solid and scientific evidence. Device manufacturers often provide scant, if any, objective data to support claims such as skin tightening, reduced pain, and improved aesthetic results.

Liposuction patients should be assessed like any other surgical patient. This includes a complete preoperative evaluation, with particular attention to anything that might predispose the patient to complications, being essential to avoid unwanted occurrences. When selecting the most appropriate techniques for each individual patient, the surgeon must consider several factors, including the anticipated liposuction volume, the number of unrelated procedures, the treatment sites, the anesthesia route, the facility type, and the patient's overall health status. As with any surgical procedure, the teaching and learning process, as well as surgical skills, are essential, not only for the surgeon but for the entire surgical team, to decrease complications rates.

Appropriate postoperative management of the liposuction patient is critical for achieving the best possible outcomes. To this end, a qualified staff is essential for providing the appropriate post-anesthesia and postoperative care. Managing the fluid and electrolyte balance, treating pain, and monitoring for complications are important duties, particularly in large-volume cases. When performed by a surgeon with knowledge of the physiologic implications of this surgery, liposuction can be a safe procedure that results in significant patient satisfaction.

\section{References}

1. Stephan PJ, Kenkel JM (2010) Updates and advances in liposuction. Aesthet Surg J 30: 83-97.

2. Kesselring UK (1983) Regional fat aspiration for body contouring. Plast Reconstr Surg 72: 610-619.

3. Mann MW, Palm MD, Sengelmann RD (2008) New advances in liposuction technology. Semin Cutan Med Surg 27: 72-82.

4. Allen Gabriel, Mary Katherine Gingrass (2014) Large Volume Liposuction Safety and Indications. CME \& Education, Medscape.

5. Haeck PC, Swanson JA, Gutowski KA, Basu CB, Wandel AG, et al. (2009) Evidence-based patient safety advisory: liposuction. Plast Reconstr Surg 124: 28S-44S.

6. Heymans O, Castus P, Grandjean FX, Van Zele D (2006) Liposuction: review of the techniques, innovations and applications. Acta Chir Belg 106: 647-653.

7. Atiyeh B, Costagliola M, Illouz YG, Dibo S, Zgheib E, et al. (2015) Functional and Therapeutic Indications of Liposuction: Personal Experience and Review of the Literature. Ann Plast Surg

8. Rohrich RJ, Beran SJ (1999) Is liposuction safe? Plast Reconstr Surg 104: 819-822.

9. Alan Matarasso, Steven ML (2003) Evidence-Based Medicine: Liposuction. Plast Reconstr Surg 132: 1435-1441.

10. B Sommer, G Sattler (2007) Indications and contraindications for liposuction. Textbook of Liposuction. Informa UK Ltd.

11. Mann MW, Palm MD, Sengelmann RD (2008) New advances in liposuction technology. Semin Cutan Med Surg 27: 72-82.

12. Sood J, Jayaraman L, Sethi N (2011) Liposuction: Anaesthesia challenges. Indian J Anaesth 55: 220-227.

13. M. Beaussier (20101) Safety Of Epidural Anaesthesia. Abstracts Of The XXIX Annual European Society Of Regional Anaesthesia Congress. Regional Anesthesia And Pain Medicine 5 Volume 35, Number 5, September-October 2010.

14. Lakshyajit DD (2008) Liposuction. Indian J Anaesth 41 (Suppl): S27 S40.

15. Klein JA (1990) The tumescent technique. Anesthesia and modified liposuction technique. Dermatol Clin 8: 425-437.

16. A Fasciolo, A Maioli (2010) Tumescent Anaesthesia And Unconscious Sedation For Liposuction: Our Experience. Abstracts Of The XXIX Annual European Society Of Regional Anaesthesia Congress 2010. Regional Anesthesia And Pain Medicine \& Volume 35, Number 5, September-October 2010.

17. Iverson RE, Lynch DJ; American Society of Plastic Surgeons Committee on Patient Safety (2004) Practice advisory on liposuction. Plast Reconstr Surg 113: 1478-1490.

18. Klein JA (1990) Tumescent technique for regional anesthesia permits lidocaine doses of $35 \mathrm{mg} / \mathrm{kg}$ for liposuction. J Dermatol Surg Oncol 16: 248-263.

19. Habbema L (2009) Safety of liposuction using exclusively tumescent local anesthesia in 3,240 consecutive cases. Dermatol Surg 35: 1728-1735.

20. Rubin JP, Bierman C, Rosow CE, Arthur GR, Chang Y, et al. (1999) The tumescent technique: the effect of high tissue pressure and dilute epinephrine on absorption of lidocaine. Plast Reconstr Surg 103: 990-996.

21. Paik AM, Daniali LN, Lee ES, Hsia HC (2015) Local anesthetic use in tumescent liposuction: an American Society of Plastic Surgeons survey. Ann Plast Surg 74: 145-151.

22. Tabbal GN, Ahmad J, Lista F, Rohrich RJ (2013) Advances in liposuction: five key principles with emphasis on patient safety and outcomes. Plast Reconstr Surg Glob Open 1: e75.

23. Rohrich RJ, Leedy JE, Swamy R, Brown SA, Coleman J (2006) Fluid resuscitation in liposuction: a retrospective review of 89 consecutive patients. Plast Reconstr Surg 117: 431-435.

24. Grazer FM, de Jong RH (2000) Fatal outcomes from liposuction: census survey of cosmetic surgeons. Plast Reconstr Surg 105: 436-446.

25. Cárdenas-Camarena L1 (2003) Lipoaspiration and its complications: a safe operation. Plast Reconstr Surg 112: 1435-1441. 
26. Grazer FM, de Jong RH (2000) Fatal outcomes from liposuction: census survey of cosmetic surgeons. Plast Reconstr Surg 105: 436-446.

27. Haeck PC, Swanson JA, Gutowski KA, Basu CB, Wandel AG, et al. (2009) Evidence-based patient safety advisory: liposuction. Plast Reconstr Surg 124: 28S-44S.

28. Trott SA, Beran SJ, Rohrich RJ, Kenkel JM, Adams WP Jr, et al. (1998) Safety considerations and fluid resuscitation in liposuction: an analysis of 53 consecutive patients. Plast Reconstr Surg 102: 2220-2229.

29. Fernando Fabricio Franco, Rafael de Campos Ferreira, Basso (2012) Complications of classical liposuction performed for cosmetic purposes. Rev Bras Cir Plast 27: 135-40.

30. Paul D. Stein, Abdo Y. Yaekoub (2008) Fat Embolism Syndrome. Am J Med Sci 336: 472-477.

31. Amandeep G, Charles S. Reilly (2007) Fat Embolism. Contin Educ Anaesth Crit Care Pain 7: 148-151

32. El-Ali KM, Gourlay T (2006) Assessment of the risk of systemic fat mobilization and fat embolism as a consequence of liposuction: ex vivo study. Plast Reconstr Surg 117: 2269-2276.

33. Wang HD, Zheng JH, Deng CL, Liu QY, Yang SL (2008) Fat embolism syndromes following liposuction. Aesthetic Plast Surg 32: 731-736.

34. Zeidman M, Durand P, Kundu N, Doumit G (2013) Fat embolism after liposuction in Klippel-Trenaunay syndrome. J Craniofac Surg 24: 1319-1321.

35. Fourme T, Vieillard-Baron A, Loubières Y, Julié C, Page B, et al. (1998) Early fat embolism after liposuction. Anesthesiology 89: 782-784.
36. Ross RM, Johnson GW (1988) Fat embolism after liposuction. Chest 93: 1294-1295.

37. Payer J Jr, Ziak P, Fedeles J Jr, Brazdilova K, Fedeles J (2013) Changes in metabolic syndrome parameters after liposuction. Bratisl Lek Listy 114: 349-352.

38. Pacholczyk M, Ferenc T, Kowalski J (2008) [The metabolic syndrome. Part II: its mechanisms of development and its complications]. Postepy Hig Med Dosw (Online) 62: 543-558.

39. Jose Antonio Robles-Cervantes, La zaro Cardenas-Camarena (2004) Modification of Insulin, Glucose and Cholesterol Levels in Nonobese Women Undergoing Liposuction: Is Liposuction Metabolically Safe? Ann Plast Surg 52: 64-67.

40. Esposito K, Giugliano G, Scuderi N, Giugliano D (2006) Role of adipokines in the obesity-inflammation relationship: the effect of fat removal. Plast Reconstr Surg 118: 1048-1057.

41. Matarasso A, Kim RW, Kral JG (1998) The impact of liposuction on body fat. Plast Reconstr Surg 102: 1686-1689.

42. Eric Swanson (2011) Prospective Clinical Study Reveals Significant Reduction in Triglyceride Level and White Blood Cell Count after Liposuction and Abdominoplasty and No Change in Cholesterol Levels. Plast Reconstr Surg 128: 182e.

43. Jae-Ho Jeong (2014) Recent Advancements in Autologous Fat Grafting. Arch Aesthetic Plast Surg 20:3. 